\title{
Disruptive life event or reflexive instrument? On the regulation of hospital mergers from a quality of care perspective
}

\author{
David de Kam, Marianne van Bochove and Roland Bal \\ Erasmus School of Health Policy and Management, Erasmus University Rotterdam, \\ Rotterdam, Netherlands
}

\begin{abstract}
Purpose - Despite the continuation of hospital mergers in many western countries, it is uncertain if and how hospital mergers impact the quality of care. This poses challenges for the regulation of mergers. The purpose of this paper is to understand: how regulators and hospitals frame the impact of merging on the quality and safety of care and how hospital mergers might be regulated, given their uncertain impact on quality and safety of care. Design/methodology/approach - This paper studies the regulation of hospital mergers in The Netherlands. In a qualitative study design, it draws on 30 semi-structured interviews with inspectors from the Dutch Health and Youth Care Inspectorate (Inspectorate) and respondents from three hospitals that merged between 2013 and 2015. This paper draws from literature on process-based regulation to understand how regulators can monitor hospital mergers.

Findings - This paper finds that inspectors and hospital respondents frame the process of merging as potentially disruptive to daily care practices. While inspectors emphasise the dangers of merging, hospital respondents report how merging stimulated them to reflect on their care practices and how it afforded learning between hospitals. Although the Inspectorate considers mergers a risk to quality of care, their regulatory practices are hesitant.

Originality/value - This qualitative study sheds light on how merging might affect key hospital processes and daily care practices. It offers opportunities for the regulation of hospital mergers that acknowledges rather than aims to dispel the uncertain and potentially ambiguous impact of mergers on quality and safety of care.
\end{abstract}

Keywords Governance, Quality healthcare, Mergers

Paper type Research paper

\section{Background}

Like many western countries (Angeli and Maarse, 2012; Bazzoli et al., 2004), The Netherlands has seen its share of hospital mergers the past decades, bringing back the number of hospitals from 243 in 1978 to 79 in 2016 (den Hartog et al., 2013; Dutch Ministry of Health, n.d.; Roos, 2018). The continued consolidation of the Dutch hospital market played out against the backdrop of sustained public and political debate on the desirability of mergers (Postma and Roos, 2016) and throughout the restructuring of the Dutch healthcare market from 2005 onwards, to make the sector more competitive (Helderman et al., 2005; Schut and Varkevisser, 2017). Mergers, we know, are challenging organisational processes that can have "multilayered and complex" effects on the services provided by organisations (Fulop et al., 2005, p. 120). In hospitals, those effects can impact the quality and safety of care provided. But, we

(C) David de Kam, Marianne van Bochove and Roland Bal. Published by Emerald Publishing Limited. This article is published under the Creative Commons Attribution (CC BY 4.0) licence. Anyone may reproduce, distribute, translate and create derivative works of this article (for both commercial and noncommercial purposes), subject to full attribution to the original publication and authors. The full terms of this licence may be seen at: http://creativecommons.org/licences/by/4.0/legalcode

We are thankful to all our respondents who so generously shared their ideas and time with us.

Funding: The research that this paper is based on was supported by ZonMw, the Dutch organisation for Health Research and Development, project number 80-83905-98-00. 
JHOM 34,4

know little about if and, in particular, how hospital mergers might impact the quality and safety of care. For one, studies that report on how hospitals perform on a set of quality indicators pre- and post-merger, deliver inconsistent and inconclusive results (Gaynor and Town, 2012; Hayford, 2012; Ho and Hamilton, 2000; Mutter et al., 2011; Romano and Balan, 2011; Vogt and Town, 2006). Also, they often offer little explanation as to how the effects observed might have come about. Second, from the literature that looks into the impact of merging on organisational processes and structures, we learn that mergers might have unforeseen, destabilising effects (Dunbar, 2011; Fulop et al., 2005; Gaynor et al., 2012). But, how those effects impact the quality and safety of care is left unaddressed. It is uncertain, in other words, how any merger might affect the quality and safety of care. While this an interesting question to consider in its own right - and we will - it is also a pressing question for regulators tasked to regulate hospital mergers and to assess how mergers might impact the quality and safety of care. Mergers between hospitals, for regulators, pose a challenge of "regulatory uncertainty" - where it is uncertain how merging might affect the quality and safety of care as well as how mergers might best be regulated (Gilad, 2010; Sabel et al., 2018). In our qualitative study, we focus on three hospital mergers in The Netherlands to answer the following questions (1) How do regulators and hospitals frame the impact of hospital mergers on the quality and safety of care? and (2) How can hospital mergers be regulated given, their uncertain impact on quality and safety of care?

In the next section of this paper, we describe what we know about the impact of hospital mergers and describe a process-based approach to regulation, developed by Gilad (2010), that can serve as a framework for understanding the impact of hospital mergers and how they might be regulated. Following that, we will describe the research methods used to provide an answer to the questions formulated above and present our findings, which we reflect further on in the discussion section of this paper.

\section{On hospital mergers, quality of care and regulation}

In the shadow of literature on the relationship between mergers and efficiency (Harris et al.,2000; Kjekshus and Hagen, 2007), prices and costs of services (Carey et al., 2011; Hutchings et al., 2003) and competition and market power (Haas-Wilson and Garmon, 2011; Schmid and Ulrich, 2013; Schmid and Varkevisser, 2016; Varkevisser and Schut, 2012), several studies look into the effect of mergers on quality of care (Beaulieu et al., 2020; Dutch Authority for Consumers and Markets, 2016; Hayford, 2012; Ho and Hamilton, 2000; Mutter et al., 2011; Romano and Balan, 2011). These studies operationalise the quality of care by comparing a set of quality indicators pre- and postmerger, testing for differences. When reviewing what the effects of hospital mergers on quality of care are, it proves difficult to say if mergers impact the quality of care positively or negatively (Gaynor and Town, 2012; Vogt and Town, 2006). Although some studies find either positive or negative effects, most studies have difficulties identifying effects in either one direction, and often the different quality indicators selected to operationalise the quality of care point in different directions (Gaynor et al., 2012; Ho and Hamilton, 2000; Mutter et al., 2011). The cautious consensus among such studies is that there is no evidence that mergers improve the quality of care. Studying the performance of hospitals merged between 2009 and 2013 in the USA, Beaulieu et al. conclude that "[their] findings challenge arguments that hospital consolidation, which is known to increase prices, also improves quality" (Beaulieu et al., 2020, p. 58).

While most studies report on the American system or that of the UK, recently, the Dutch Competition Authority (The Netherlands Authority for Consumers and Markets) commissioned a study into the effects of hospital mergers on the quality of care in The Netherlands (Dutch Authority for Consumers and Markets, 2016). This study's findings, studying 14 hospital mergers between 2007 and 2013, are in line with those found elsewhere; while some hospitals improved on some quality indicators after merging, they scored worse on others. As the quality of care is impacted by much more that mergers alone, definitive conclusion could not be drawn 
(Dutch Authority for Consumers and Markets, 2016). The study noted that the impact of a merger on the quality and safety of care is "intermediate"; it can impact hospital processes and structures in positive and negative ways (e.g. merging can offer increased opportunities to train medical professionals, as well as distract from the primary process), but it is unclear if this eventually affects hospital outcomes (Dutch Authority for Consumers and Markets, 2016).

Few studies have empirically studied how mergers affect structures and processes in healthcare organisations (Dunbar, 2011; Fulop et al., 2005; Gaynor et al., 2012). Fulop et al. study mergers from an organisational change perspective and argue that "mergers are based on simplistic notions of organisational change" (2005, p. 120) that fail to account for how a merger might impact organisations and its personnel in unintended ways. A merger, according to Fulop et al. is a complex, layered organisational restructuring process that brings about "a period of (...) intense introspection" (2005, p. 129). Mergers often fail to deliver on their promises of services improvement (Ahgren, 2008; Fulop et al., 2005; Gaynor et al., 2012; Rohde and Torvatn, 2017) while struggling to steer clear of dysfunctional effects, "including loss of focus on services, delays in improvements, loss of organisational memory and inter-organisational relationships, and difficulties in transferring good practice" (Dunbar, 2011). Fulop et al. write that their respondents reported that "loss of managerial focus on services during the merger had harmed patient care (. . .) and that the merger made services worse" - but fail to elaborate on why or to what extent this was the case (2005, p. 127). A merger is not just a means to the end of reorganising existing organisational structures, but is itself a complex "evolutionary process" that leaves its mark on organisations (Fulop et al., 2005, p. 129). Haas et al. in a paper on healthcare expansions in the USA, report that mergers can create risks to patient safety as they inevitably introduce changes to patient populations, organisational infrastructure and/or clinician practice settings (Haas et al., 2018). While the literature discussed so far relates the process of merging to quality and safety of care, it fails to make specific how or under what conditions merging has effects on the quality and safety of care. It is exactly this that is necessary from a regulatory perspective.

Regulation entails "the sustained and focused attempt to alter the behaviour of others according to defined standards and purposes with the intention of producing a broadly identified outcome or outcomes, which may involve mechanisms of standard-setting, information-gathering and behaviour-modification" (Black, 2002, p. 26). This definition addresses both the goal and focus of regulation (altering behaviour to produce valued outcomes), and some of the instruments regulators might use to achieve that goal (like standard-setting). In our complex and fast-changing world, various scholars argue regulators face challenges that prescriptive regulation - regulator's traditional mode of operation - is illequipped to deal with (Baldwin et al., 2012; Gilad, 2010; Gunningham, 2012). Prescriptive regulation assumes that it is clear what behaviour of regulatees is expected to ensure a valued outcome (Baldwin et al., 2012). In such cases, regulators can impose standards that regulatees ought to comply with. Yet, when there is no certainty about what kind of regulatee behaviour produces the identified outcome, or when what the outcome should be is subject to debate, prescriptive regulation is of little help (Baldwin et al., 2012; Gilad, 2010; Gunningham, 2012). That is why, Gilad writes, contemporary regulators "[experiment] with regulatory arrangements that allow regulated organizations flexibility to tailor regulation to their individual circumstances, while holding them accountable for the adequacy and efficacy of their internal control systems" (Gilad, 2010, p. 485). Rather than strictly prescribing what regulatees should and should not do, regulators expect regulatees to "self-regulate", to design, manage, monitor and (re)evaluate the quality of their own services (Gilad, 2010). In this "process-oriented" approach to regulation, regulation can target one of the three distinct organisational tiers (Table 1). The first-tier concerns an organisation's key production processes. The second-tier concerns an organisation's control systems through which it monitors its first-tier production processes. The third-tier concerns an organisation's 


\section{JHOM 34,4}

Tier of

regulation

Focus of regulation

Regulatory standards

Type of regulation

\begin{tabular}{|c|c|c|c|c|}
\hline & First & $\begin{array}{l}\text { Organisations' core } \\
\text { production and } \\
\text { operations }\end{array}$ & $\begin{array}{l}\text { Prescribed actions, output specifications } \\
\text { or principles that control and constitute } \\
\text { firms' production processes }\end{array}$ & $\begin{array}{l}\text { Prescriptive or } \\
\text { outcome-oriented } \\
\text { regulation }\end{array}$ \\
\hline 492 & Second & $\begin{array}{l}\text { Organisations' } \\
\text { compliance systems }\end{array}$ & $\begin{array}{l}\text { System-oriented specifications that direct } \\
\text { organisations' governance and control of } \\
\text { their compliance with first-tier } \\
\text { specifications }\end{array}$ & $\begin{array}{l}\text { Controls-based } \\
\text { regulation }\end{array}$ \\
\hline $\begin{array}{l}\text { Table } \mathbf{1} \text {. } \\
\text { Tiers of regulation } \\
\text { (Gilad, 2010, p. 490) }\end{array}$ & Third & $\begin{array}{l}\text { Organisations' } \\
\text { self-evaluation }\end{array}$ & $\begin{array}{l}\text { Process-oriented specifications that guide } \\
\text { organisations' analysis of the risks that } \\
\text { their operations pose to regulatory } \\
\text { objectives and their setting of first- and } \\
\text { second-tier specifications }\end{array}$ & $\begin{array}{l}\text { Process-oriented } \\
\text { regulation }\end{array}$ \\
\hline
\end{tabular}

self-evaluative activities and its evaluation and (re)design of its first-tier operations and second-tier controls (Table 1). For example, a regulator might monitor: if a hospital complies with the mandated use of surgical safety checklists (first-tier), what processes or systems the hospital has put in place to ensure that these checklists are used (second-tier) and on how the use of checklists is tied to and embedded in other safety practices or how the hospital deals with situations where professionals deviate from (using) the checklist (third-tier).

While Gilad developed the three regulatory tiers in a review article that spans a range of regulatory (process-based) approaches, for us, it provides a framework for understanding where our respondents believe the impact of a merger on the quality and safety of care is located (in terms of the three tiers). We can use it to wonder how a merger might impact a hospital's key processes or its capacity to self-evaluate those processes. Also, if offers a way to think about how mergers might be regulated. Gilad notes that process-based regulatory approaches do not do away with oversight on the first or second tier. Rather, these new regulatory approaches tend to be layered onto already existing regulatory approaches (Gilad, 2010; van de Bovenkamp et al., 2014). When it comes to hospital mergers, we can wonder how the Inspectorate can attend to how the process of merging impacts hospital performance on all three tiers.

\section{Methods}

To answer our research questions, we adopted a qualitative research design.

Our paper is based on 30 semi-structured interviews that we conducted throughout three phases of our research as well as document analysis. First, we browsed newspapers, healthcare management websites and blogs discussing mergers. Through our reading, we got a clear image of the discussions about mergers in relation to the quality of care in The Netherlands. We also in this way identified six individuals that were often asked to weigh in on these discussions, given their experience as (former) organisational change consultants or (former) hospital directors or regulators. We approached these individuals through email, asking them to participate in our study. All were willing to be interviewed. In these interviews $(n=6)$, we tried to better understand how the process of merging might impact hospital processes that impact the quality and safety of care. Second, we approached the regulatory agencies involved in assessing and approving a merger in The Netherlands (Box 1). We conducted interviews with inspectors and other employees of these agencies to understand how hospital mergers are currently regulated and what issues they encounter. We were specifically interested in how the Dutch Health and Youth Care Inspectorate (further: Inspectorate), responsible for overseeing the quality and safety of care regulates hospital 


\section{Box 1. Hospital merger control in The Netherlands}

All mergers in The Netherlands require approval by The Netherlands Authority for Consumers and Markets (Competition Authority) if both organisations together generate an annual revenue of $€ 150 \mathrm{~m}$ or more globally and if at least two of the merging organisations each has an annual revenue of $€ 30 \mathrm{~m}$ or more in The Netherlands. The Competition Authority oversees the Competition Act and is the regulatory body that either clears or prohibits mergers. The decision of the Competition Authority to clear or prohibit a merger boils down to the question of whether a merger is (expected to be) anti-competitive. Since 2014, mergers between healthcare organisations also require approval of the Dutch Healthcare Authority (Healthcare Authority). The Healthcare Authority evaluates if the merger poses a threat to access to vital hospital services (such as emergency care) in the region. The Inspectorate enters the stage within the evaluation of the Healthcare Authority, because the Healthcare Authority can consult the Inspectorate and inquire how the quality of care might be impacted by the merger. The Competition Authority, weighing both the Healthcare Authority's and Inspectorate's input, clears or prohibits the merger. The Competition Authority has approved 26 of the 27 proposed hospital mergers since 2004. Aside from mergers, the Inspectorate is charged with overseeing the quality of care provided by healthcare organisations and professionals throughout the country, which has to be up to par, regardless of possible organisational concerns they might face (Inspectorate, 2016)

mergers, so that most of the interviews we conducted in this round $(n=8)$, were conducted with employees of the Inspectorate. Third, we purposively selected and approached three recently merged hospitals, spread throughout the country and varying in size (Table 2). Through the board members of these hospitals, we gained access to all three hospitals, and in each hospital, interviewed (at least): the executive director, the chair of the medical staff, the chair of the nursing committee, the chair of the patient committee and a quality of care manager. We conducted 16 interviews in the hospitals.

Interviews were conducted between September 2015 and March 2016. Most interviews were conducted by the first (DdK) and second (MvB) author together, and in some, the third author participated. Interviews lasted for $61 \mathrm{~min}$ on average. Topic guides, which were prepared by (A) and discussed among all authors, structured the interviews. With consent of the respondents, interviews were taped and transcribed verbatim afterwards. The transcribed interviews were stored in accordance to the research data management (RDM) principles of our institution, and data were only shared among and accessible to the authors of this paper. As all the authors also conducted the interviews, transcripts were not

\begin{tabular}{lccc}
\hline Characteristics & Merger 1 & Merger 2 & Merger 3 \\
\hline Approval Competition Authority & 2012 & 2014 & 2012 \\
Administrative merger* & 2013 & 2015 & 2013 \\
Legal merger* & 2015 & 2017 & 2016 \\
Number of beds** & $555 / 551$ & $923 / 209$ & $545 / 424$ \\
Distance between hospitals*** & $7 \mathrm{~km}$ & $25 \mathrm{~km}$ & $8 \mathrm{~km}$
\end{tabular}

Note(s): *Dutch law differentiates between mergers based on the level of integration between merging parties. In the case of an administrative merger, both hospitals (as distinct legal organisational entities) continue to exist but are governed by a central body (often a new legal entity that governs the two merged hospitals as subsidiaries). In the case of a legal merger, the two legally distinct hospitals merge and continue as a single legal organisational entity. Often, Dutch hospitals first merge on an administrative level to later merge into a single organisation

**As self-reported by the separate hospitals to the Dutch Ministry of Health, in the year of approval for the merger by the Competition Authority (Dutch Ministry of Health, n.d.)

***Obtained through Google Maps, walking distance, rounded off 
JHOM 34,4

494

anonymised or pseudonymised during data analysis. Anonymisation of cited excerpts from the interviews was done during writing of the manuscript.

To analyse our data, we opted for an abductive approach (Tavory and Timmermans, 2014), meaning that we came to understand our data as we iteratively moved back and forth between our data and theory. We coded our research data openly and through an indicative analytic approach identified emerging themes (Green and Thorogood, 2018), but we turned towards theory to better understand the themes we identified. Atlas.ti was used to facilitate the coding process. (A) conducted the initial coding process and generated a multitude of codes. These codes were then discussed among (A) and (B), as we sought to identify the relationships between codes. The identification and analysis of themes in light of the theory introduced was discussed between all three authors through a series of meetings.

\section{Results}

Our results are ordered into three parts. First, we present how our respondents framed the quality and safety of care as something that needs attending to. Second, we present how merging can impact key hospital processes as care practices are reorganised. Third, we present how the Inspectorate regulates mergers as well as how hospital respondents experienced the Inspectorate's regulation.

\section{Attending to the quality and safety of care in the process merging}

The Inspectorate conceives of a merger as a big organisational change that may negatively affect the quality of care. "In periods of organisational change, the risks of blind spots and errors in healthcare organisations is greater: organisations are less attentive to the continuity and quality of care" (Inspectorate, 2015). Healthcare inspectors voice similar concerns. "Mergers pose a risk to quality of care in hospitals, because attention is diverted away from the primary process" (Inspector 2). "Mergers sap all energy out of quality and safetyeverything else goes on hold. So much has to be invested in merging that improving quality and safety for the patient ... there's no time for that" (Inspector 1). Inspectors colloquially refer to a merger as a life event. Life events are "very drastic", disruptive events that shake up and (temporarily) preoccupy an organisation - like merging, facing financial problems, introducing a new electronic patient record or top-level management changes are referred to by inspectors as life events. In the case of a life event, "it becomes really hard for organisations to meet required standards" (Inspector 1).

Hospital respondents acknowledge that merging is demanding. "The merger costs a lot of time and energy. Not all specialists can do their consults or surgeries, because they have to be part of discussions about how we'll work together in the new operating theatre" (Medical specialist 3). "The merger asks a lot. (...) We have to, on the one hand, continue patient care, and on the other, involve professionals in things like aligning protocols" (Quality of care manager 2). Multiple respondents granted that, during the merger, they were "turned inwards". "Really, we focus on ourselves and less on developments outside our own hospital. So, patient self-management is a thing right now-and that'll come, but it's not our main concern now" (Nursing association chairs 1). A medical specialist noted that when occupied with the merger, innovation suffers.

The quality of care, our respondents agreed, requires constant work and attention, and a merger might pose a risk to the quality of care because it could (temporally) disrupt that attention. Hospital respondents, however, also challenged the idea that merging and attending to the quality of care are two disparate things - where attending to one prevents you from attending to the other. Most hospital respondents argued that because they were aware of the risks a merger might pose to the quality of care, the quality of care received a lot 
of attention. "A merging organisation is incredibly self-critical. Quality of care has never gotten the attention it's getting now that we're merging. It's great" (Quality of care adviser 1). Additionally, respondents told us that hospitals consistently face issues not directly related to quality of care. "We're always reorganising hospitals. If there's a new guideline, new medication, new operating procedure-you have to reorganise processes" (Medical specialist 1). The attention of a hospital is never exclusively directed at the quality of care and non-merging hospitals equally deal with organisational change, our respondents argued. Mergers, according to hospital respondents, are no different from normal change routines, but can be seen as an intensification of those routines. Whilst the Inspectorate, thus, frames mergers as a life event that takes attention away from the quality of care, hospital respondents argue that quality of care is foregrounded during the merging process, albeit acknowledging that innovation temporarily comes to a halt.

\section{Reorganising care practices}

Typically, when hospitals in The Netherlands merge, the hospitals become separate locations of the same hospital organisation, and care services are divided between them. Some services might exist on both locations, while others might be exclusive to one location. The hospitals we studied asked the personnel to work at both locations. All respondents reported that this way of working poses risks. Ironing out differences between locations - in terms of protocols, electronic patient records, medical equipment, etc. - is the main priority in a merger when the personnel works at both locations, respondents argued. Doing so is "a huge amount of work" that is challenging both in the effort it takes and because these differences are ingrained in the nuts and bolts of organisational processes. A consultant told of two hospitals, "that both had four-bed rooms. In one hospital they counted those beds clockwise, in the other, counterclockwise ... That's dangerous with medications" (Consultant 1). Hospitals try to identify such differences by setting up "transitional teams" or "protocol committees" tasked with aligning these differences, often on a departmental level. But, even with a committee in place, it can be difficult to foresee risks.

If you don't program an infusion pump correctly, a patient receives a wrong dose. And here they use different pumps than on the other location, but nurses work on both locations. We had not identified this as risk prior to relocating services and personnel ... Its something you run into (Quality of care adviser 1).

Really, if you're quality-minded, you'll have to put on the brakes at times. You'll identify a safety issue, meaning you cannot just merge two departments or disciplines, because different protocols need to be aligned first (Medical specialist 2).

As personnel and care practices are relocated as part of the merging process, a merger intervenes in and potentially disrupts many interwoven processes. "Everything's connected! The moment you relocate care practices, so much changes-going back to the beds you use, the infusion pumps, administering medication ..." (Quality of care manager 2). A merger challenges the historically rooted, everyday logic of "the way we do things here", that can go back to the smallest of details. How those details might come to matter in everyday care practices and the outcomes of those practices is hard to foresee.

Because of the work it takes to address and align these differences in how hospitals work and the risks that occur when it is not done properly, the Inspectorate considers a merger a considerable risk to the quality of care.

Protocols, ordering medication, it's all different. [Merging] is building an entirely new organisation - on top of an already busy, regular workload ... All kinds of risks to patient care emerge (Inspector 4). 
JHOM 34,4

496
Whilst hospital respondents do not deny these risks, they also point out how having to align protocols and rethinking why you do things a certain way offers learning opportunities. A director experienced the merger as a "leap forward", as it forced them to "critically question each other: "Why do you this in this way? Can't you also do it like this?" (Executive director 2). Respondents present a merger as a reflexive instrument that, in its potential to disrupt the historically rooted "way we do things", offers a valuable opportunity to revaluate current care practices and improve upon them. In merging, respondents claim, they can learn from the other hospital. "On one location, when elderly are brought in through emergency care, a geriatrician assesses if someone is demented or suffers a delirium. That's a practice we'll implement here too" (Quality of care adviser 1). While the idea that in comparing care practices, hospitals can evaluate and implement best practices was voiced by multiple respondents, this is no given, and the question of what a best practice is, is not beyond organisational concerns or power relationships. In the merger between a large and a small hospital, this was clearly apparent:

Chances are, our way of working will be dominant, just because we're bigger. It's way more work to implement a best practice of theirs over here, than the other way around, just because they're a much smaller hospital. I wonder if their best practices are really seen as such, if they are implemented here. (...) I do not think it likely (Quality of care manager 3).

Both inspectors and hospital respondents identify risks associated with how, in merging, care practices are relocated and healthcare professionals are asked to work on both hospital locations. What becomes apparent is that the process of merging, for both inspectors and hospital respondents, can (temporarily) destabilise routines. Inspectors tend to emphasise the dangers of this destabilisation of practices and routines - as healthcare professionals can be confronted with different protocols or information technology (IT) systems, they are unable to access on the spot. Hospital respondents acknowledge the risks associated with redesigning practices and altering routines, as well as the time this takes, but also frame this temporary destabilisation as a productive opportunity to critically reflect on and improve one's care practices. These findings shed some light on how key processes - e.g. medication administration procedures - might be impacted as hospital merge, but rather than identifying specific processes as such, what we learn is that a merger can challenge and possibly destabilise a rooted familiarity healthcare professionals have with care processes and practices. Given this, we wonder how the Inspectorate regulates hospital mergers and how hospital respondents experience that regulation.

\section{Regulating hospital mergers}

In the regulation of mergers, the Inspectorate is involved in two stages. The first is merger control and the question if a merger should be approved, at which stage the Inspectorate can give their take on how a merger is expected to impact the quality of care (Box 1). The second entails the regulation of hospitals once the merger is approved. We discuss both.

Assessing the impact of a hospital merger on the quality of care. In assessing whether a merger should be approved, the Inspectorate is asked to weigh in on how the merger might impact the quality of care. Inspectors perceive this as an impossible task. "The expectation is that we can predict [the impact of a merger on quality of care], (...) but we cannot" (Inspector 2). A policy adviser told us, "we have an image of hospital A and of hospital B, but that does not become [image] C, when you merge those. That becomes a new image", underscoring the unpredictable impact of a merger. Faced with what the Inspectorate considers the impossible task of "predicting" the impact a merger will have on the quality of care, the Inspectorate refrains from giving their take on a merger and is de facto not involved in merger control. The Inspectorate's self-proclaimed inability to predict the impact of future 
mergers stems from the conviction that the impact of a hospital merger on the quality of care is uncertain, and that as each merger is thought to have its own unique dynamic, past mergers do not provide the Inspectorate with knowledge about how a future merger might play out. The Inspectorate opts out of being meaningfully involved during hospital merger assessment so that the risks a merger might pose to the quality of care - detracting attention or destabilising familiarity with care processes and practices - do not feature in the decision to approve of prohibit a merger.

Regulating hospital mergers after approval. Although inspectors conceive of mergers as risk to the quality of care - in the attention it requires, detracting from the attention (needed) for the quality of care and as hospitals relocate care practices and personnel - inspectors explained that as hospitals merge, this, in itself, is no reason to alter or intensify regulation. Having said that, various inspectors monitor merging hospitals more closely "behind the scenes, so that we can intervene before performance deteriorates" (Inspector 3). The Inspectorate possesses no formal regulatory tool based on which they monitor merging hospitals differently than other hospitals. Consequently, inspectors take different approaches. While some inspectors wait for "really hard signals - more reported incidents, more complaints-that indicate quality of care is under pressure" (Inspector 2), other inspectors take a different approach.

I visited both hospital locations, unannounced, to see how both were doing on a specific theme. I found that while they'd already relocated care practices, they had not yet aligned protocols and personnel was insufficiently trained at the new location. I said, "You cannot continue like this; you have to train personnel and align protocols and systems before you allow personnel to work at the other location!" (Inspector 4)

Despite the way inspectors previously expressed hospital mergers to be disruptive, discontinuous life events, the Inspectorate's regulatory practices do not (formally) change in response to an announced or approved merger - only to the extent that individual inspectors might opt to "follow" hospitals more closely.

How hospital respondents experienced the Inspectorate's regulation. Multiple respondents from hospitals noted that the Inspectorate was "absent" as they prepared the merger. They expressed the expectation that the Inspectorate would be more present after plans for merging with another hospital were announced. In fact, many respondents thought it was a shame that the Inspectorate left the hospitals to their own devices and proposed ways in which the Inspectorate could be meaningfully involved in merger assessment - without the Inspectorate feeling the pressure to predict the impact of a merger.

The Inspectorate should look closely at the relocation of professionals and care practices-that's when things really change. Look at key processes: patient-identification, medication processes, operating theatre procedures, handovers (Quality of care manager 2).

The Inspectorate could say "In merging, we see such and such risks" and: "What care practices will you relocate?” A prospective risk analysis (...) forces you to structurally assess what might go wrong (Executive director 1).

Before a merger is approved, respondents from hospitals envision two roles for the Inspectorate. For one, the Inspectorate could critically question hospitals about how they intend to relocate care practices and professionals safely, what risks might be involved in this relocation and how they mean to address these risks. Secondly, the Inspectorate - having seen many mergers - could share its knowledge with hospitals that are preparing a merger so they might benefit.

The Inspectorate, in turn, is hesitant to take on this second role, claiming that hospitals are well-equipped to share such knowledge amongst themselves. "That is not our role. I understand why hospitals might want us to do this, but that is not our part to play; we are not 
JHOM 34,4

498 advisers, we are inspectors" (Inspectors 3). The notion that to regulate is something quite distinct from providing the parties it regulates with advice is a notion that was (often quite strongly) expressed by multiple inspectors.

Interviewer: So, you do not have an advisory role?

Inspector 2: Oh no, definitely not! We stay far away from that. Whenever the expectation that we would be able to advise hospitals [on the merging process], we back away and reiterate: "We do not advise hospitals in merger processes, nor do we judge the [merits of a] merger; that is a process between two hospitals, wherein the regulator plays no part, should play no part-unless we find that the merger negatively impacts quality of care, unless we receive signals that things go awry."

In line with how different inspectors might take different approaches to regulating hospitals after the approval of a merger, hospital respondents experienced the Inspectorate's regulatory presence differently once the merger was approved. Whereas directors from two hospitals were visited more often at the time of the merger, another director was unaware of any increased attention from the Inspectorate. "We're treated like any other hospital. I've talked with the Inspectorate about relocating care practices and showed them what we're doing. We've demonstrated what risks might emerge because of this and how we'll manage them" (Executive director 1). Other respondents from hospitals, quality managers and medical specialists would value (additional) thematic regulatory visits from the Inspectorate specifically targeting risky aspects of the merger - such as the relocation of care services, or how the hospitals ensure continued access to IT systems as personnel works at both locations. Here, too, the Inspectorate is reluctant to do so, as this, inspectors argue, would entail prioritising regulatory practices to the needs of hospitals that decided to merge, rather than to the regulatory agenda of the Inspectorate.

The Inspectorate's increased attention for merging hospitals is not formalised. That a hospital is merging is something inspectors "keep in mind" and monitor "behind the scenes", but the decision to act on that is up to individual inspectors and depends on additional criteria (more reported incidents or "other signals" that a hospital is struggling).

\section{Discussion}

We wanted to understand how the process of merging impacts the quality and safety of care through key hospital processes and what this implies for how mergers might be regulated. Hospital respondents and inspectors acknowledge that merging is an arduous organisational process. The Inspectorate primarily conceives of a merger as a risk to the quality of care, as it detracts from attention and time necessary for attending to the quality of care, and it disrupts safety routines. Hospital respondents point out that attending to the merger and attending to the quality of care are not necessarily different things. Risks to the quality of care are said to occur, especially as personnel and care practices are relocated. Hospitals organise their care practices differently and use different equipment and IT systems to support those practices. When personnel works at both locations, these differences pose risks to the quality and safety when they are not properly identified (e.g. "miscounting" beds) or not yet aligned (having to operate an unfamiliar infusion pump). Ironing out these differences is a priority and a challenge, because differences can be difficult to identify and because of the work it takes.

We were also interested in how the Inspectorate regulates hospitals mergers. While the Inspectorate states a merger might detract from attending to the quality and safety of care and it might destabilise care practices, its regulatory practices are hesitant. Prior to the approval of a merger, the Inspectorate can weigh in on how a merger might impact the quality of care, but refrains from doing so because it considers "predicting" the impact of a merger impossible. After a merger has been given the go-ahead, the Inspectorate does not monitor these hospitals differently than it does others, although some inspectors follow merging hospitals more 
intensively "behind the scenes". The uncertainty that surrounds the question of how a merger will play out hampers the Inspectorate in taking a more proactive regulatory stand. In terms of the regulatory framework of Gilad (2010), the Inspectorate conceives of the impact of a merger as possibly affecting a hospital's first-tier operations - like its medication processes. But, a merger's unpredictable character and the idea that each merger is its own unique process hamper the development of a prescriptive regulatory approach. What is interesting is that the Inspectorate, in ruling out the possibility of first-tier prescriptive regulation in response to mergers, does not explore the possibility for regulating hospital mergers on the second or third tier - that would allow for regulating the quality of the systems and self-evaluative activities of merging hospitals. Hospital respondents, meanwhile, welcome regulatory activities that requires them to account for such activities, by, for example, demonstrating how they ensure the safety of key processes as they relocate care practices.

In line with previous studies, we found that mergers initiate a period when hospitals are "turned inwards" and staying up to date with latest developments becomes challenging. Also, while implementing best practices between hospitals was cited as a reason for why mergers help improve care practices, what these "best" practices are is not evident, nor is implementing a practice elsewhere straightforward. We address a gap in the current literature, as outcome-oriented studies do not demonstrate how mergers impact the quality of care, while the process-oriented studies insufficiently trace the impact of a merger on organisational processes to the quality of care. We contribute to both literatures by demonstrating that a merger is a complex process that can intervene in interwoven care practices and uproot established, tacit ways of working. While some of its impact might be anticipated and organised for - e.g. by aligning protocols prior to relocating personnel and care practices - all the ways in which a merger might impact care practices is difficult to foresee. Allen, studying the work of nurses, notes that "[nurses] displayed a detailed understanding of the relevant activity systems in the local ecologies in which they worked, including role formats, routines, bed economy, material and psychological artefacts and temporal structures" (2015, p. 137). The manner in which nurses were able to weave together clinical and organisational knowledge depended on a (long-standing) familiarity with one's organisational surroundings (Allen, 2015). Mergers can uproot vested practices and destabilise healthcare professionals' familiarity with a hospital's "local ecology". This destabilisation is predominantly framed as a threat by the Inspectorate, while hospitals claim it is productive too. This period of temporary destabilisation is conceived of by hospitals as a chance to critically reflect and improve upon their care practices. The need to reassess one's routines and practices, hospital respondents claim, also allows for pin-pointing best practices - even if the evaluation and implementation of a best practice is not straightforward (cf. Fulop et al., 2005). The impact of a merger on the quality of care is said to be ambiguous (offering learning opportunities as well as posing dangers), potentially disruptive in unforeseen ways and emergent as many potential risks become visible only when stumbled upon. The multitiered regulatory approach we suggest below aims to be attentive to these characteristics of a merger while also providing regulators with an approach to monitor and potentially act on how hospitals manage a merger.

Many of the studies that report on the relationship between a merger and quality and safety of care do so through quantitative research designs. While these studies might indicate if merging impacts quality and safety of care, they have some disadvantages. First, these studies must assume "a merger" as a clearly bounded variable, to which potential differences in quality indicator scores pre- and post- merger might be ascribed. This, others recognise as well, is problematic (Dutch Authority for Consumers and Markets, 2016; Fulop et al., 2005). "A 'merger' is a unit of analysis with 'ambiguous boundaries' (. . ); when the process 'begins' and 'ends' is not obvious. Previous research treated 'merger' as having clear boundaries and therefore underestimated its complexity." (Fulop et al., 2005, p. 120) Second, in selecting a set 
JHOM 34,4

500 of quality indicators to assess the impact of a merger, these studies predetermine what "quality and safety of care" supposedly is. In our qualitative study, we developed a different perspective. We approached a merger as a complex organisational process with "ambiguous boundaries" that can impact a hospital's care practices. A merger, viewed as such, is a process a range of people invest in to normalise new organisational structures and routines (May and Finch, 2009). Through our semi-structured interviews, we gained an understanding of what such investments entail and how merging, as a process, can impact key hospital processes. In our design, moreover, we did not assume to know what the quality and safety of care is, or resides, but rather, have our respondents give their take on how they perceive a merger impacting quality and safety of care. We involved both the perspectives of merging hospitals as well as healthcare inspectors since regulation is a relational practice (Gilad, 2010). To regulate is to establish a relationship between a regulator and a regulatee that is directed towards a particular goal (typically a form of regulatee behaviour). To address the question of how mergers might be regulated, we therefore considered both the perspectives of regulator and regulatees. While our study design proved informative given the questions we looked to address, it also has some limitations. For one, our sample of respondents $(n=30)$ was relatively small and true anonymisation of hospitals proved difficult. This might have affected the extent to which our respondents provided socially desirable accounts of the merger process. Yet, our findings on the opportunities a merger holds and the challenges it poses (for quality and safety of care) are not dissimilar to those reported in studies that, given their data set, were better able to assure anonymity of hospitals and its employees (Fulop et al., 2005; Haas et al., 2018). Also, while we understand a merger as a complex process that people invest in over an extended period, our interviews were conducted at one moment during that period, rather than following the merger process and our respondents over time. Having said that, the strength of our study is in how it documents the impact of a merger on quality and safety of care from multiple perspectives (hospital respondents and healthcare inspectors) and how understanding those perspectives allows for thinking about an alternative way to regulate hospital mergers.

\section{Recommendations for the future regulation of mergers}

In the introduction, we wrote about the uncertain impact a merger might have on the quality and safety of care. While we shed some light about how a merger might impact the quality and safety of care - destabilising healthcare professional's familiarity with care processes and practices - it remains anything but predictable. The question is, then, how the Inspectorate might develop a regulatory approach to mergers, given their uncertain impact? Before a merger is approved, we suggest the Inspectorate requires hospitals to conduct prospective risk analyses that they report to the regulator - addressing how their plans to merge and possibly relocate care practices and personnel might impact key processes - that serves to design and possibly reorient reorganisation practices. More than exhaustively identifying all the risks a merger poses, a prospective risk analyses can serve as a heuristic exercise that challenges both hospitals and regulator to reflect on the possible impact of a merger on the quality of care and to acknowledge a merger as complex, disruptive reorganisational process, without giving in to the idea that all dysfunctional effects can be mitigated. This is a prescriptive regulatory intervention on the second and third tier, as it calls hospitals to monitor the safety and quality of their practices and the risks merging might pose to those practices, which does not require certainty about a merger's impact on care practices. It ensures hospitals focus on and think about the quality and safety of their practices in the process of merging and to install procedures that ensure that reflecting on the disruption of care and safety routines is actually done. Moreover, it can accommodate for the unique character of a merger. Hospitals have their strengths and weaknesses and the Inspectorate that generally knows about the vulnerabilities of a given hospital - can ensure that hospitals 
focus on how merging impacts their key processes. Hospitals, in other words, are expected to tailor this regulatory instrument to their own situation (Gilad, 2010). This process-based regulatory intervention does not do away with first-tier regulation - such as monitoring a hospital's performance on those "hard signals", like quality indicators, one inspector mentioned - but it can mediate what those signals mean. Documented poor performance in combination with a prospective risk analysis that does not convince the Inspectorate that a hospital is aware of the risk a merger might pose might be handled differently than if a risk analysis is indicative of a hospital's willingness and capacity to critically self-evaluate its practices. Such a regulatory approach acknowledges, rather than aims to dispel, a merger's uncertain impact on the quality and safety of care.

\section{Conclusion}

Mergers are complex reorganisational processes that can disrupt daily care practices in hospitals. In a merger, different ways of working that hospitals developed over the years meet and are challenged. While the Inspectorate emphasises how a merger poses risks to the quality of care, hospitals draw attention to the productive side of having to critically reassess current care practices. We call for regulatory practices that acknowledge the uncertain impact of a merger. Adopting a process-based regulatory approach, which requires hospitals to report on the risks merging poses to the quality and safety of their care practices, can help doing so.

\section{References}

Ahgren, B. (2008), "Is it better to be big?”, Health Policy, Vol. 87, pp. 92-99, doi: 10.1016/j.healthpol.2008. 02.001 .

Allen, D. (2015), The Invisible Work of Nurses: Hospitals, Organisation and Healthcare, Routledge Advances in Health and Social Policy. Routledge, New York.

Angeli, F. and Maarse, H. (2012), "Mergers and acquisitions in Western European health care: exploring the role of financial services organizations", Health Policy, Vol. 105, pp. 265-272, doi: 10.1016/j.healthpol.2012.02.012.

Baldwin, R., Cave, M. and Lodge, M. (2012), Understanding Regulation: Theory, Strategy, and Practice, 2nd ed., Oxford University Press, New York.

Bazzoli, G.J., Dynan, L., Burns, L.R. and Yap, C. (2004), "Two decades of organizational change in health care: what have we learned?", Medical Care Research and Review, Vol. 61, pp. 247-331, doi: 10.1177/1077558704266818.

Beaulieu, N., Dafny, L., Landon, B., Dalton, J., Kuye, I. and McWilliams, M. (2020), "Changes in quality of care after hospital mergers and acquisitions", New England Journal of Medicine, Vol. 382, pp. 51-59, doi: 10.1056/NEJMsa1901383.

Black, J. (2002), "Critical reflections on regulation", Australian Journal of Legal Philosophy, Vol. 27, pp. 1-35.

Carey, K., Burgess, J.F. and Young, G.J. (2011), "Hospital competition and financial performance: the effects of ambulatory surgery centers", Health Economics, Vol. 20, pp. 571-581, doi: 10.1002/ hec.1617.

den Hartog, M., Janssen, R., Haselbekke, B.J., Croes, R. and Klik, M. (2013), "Factors associated with hospital closure and merger: a survival analysis of Dutch hospitals from 1978 to 2010", Health Services Management Research, Vol. 26, pp. 1-8, doi: 10.1177/0951484813481768.

Dunbar, J.A. (2011), “When big isn't beautiful: lessons from England and Scotland on primary health care organisations", Medical Journal of Australia, Vol. 195, pp. 219-221.

Dutch Authority for Consumers and Markets (2016), Ziekenhuisfusies en kwaliteit van zorg: Onderzoek naar de effecten van ziekenhuisfusies op de kwaliteit van zorg, Significant, Barneveld. 
JHOM 34,4

Dutch Ministry of Health (2018), Aantal Instellingen Voor Medisch Specialistische Zorg, Dutch Ministry of Health.

Fulop, N., Protopsaltis, G., King, A., Allen, P., Hutchings, A. and Normand, C. (2005), "Changing organisations: a study of the context and processes of mergers of health care providers in England", Social Science and Medicine, Vol. 60, pp. 119-130, doi: 10.1016/j.socscimed.2004.04.017.

Gaynor, M. and Town, R. (2012), The Impact of Hospital Consolidation-Update, the Synthesis Project, Robert Wood Johnson Foundation.

Gaynor, M., Laudicella, M. and Propper, C. (2012), "Can governments do it better? Merger mania and hospital outcomes in the English NHS", Journal of Health Economics, Vol. 31, pp. 528-543, doi: 10.1016/j.jhealeco.2012.03.006.

Gilad, S. (2010), "It runs in the family: meta-regulation and its siblings: meta-regulation and its siblings", Regul. Gov, Vol. 4, pp. 485-506, doi: 10.1111/j.1748-5991.2010.01090.x.

Green, J. and Thorogood, N. (2018), Qualitative Methods for Health research, 4th ed., Introducing Qualitative Methods Series - IQM, SAGE Publications, Thousand Oaks, CA.

Gunningham, N. (2012), "Regulatory reform and reflexive regulation: beyond command and control", in Brousseau, E., Dedeurwaerdere, T. and Siebenhüner, B. (Eds), Reflexive Governance for Global Public Goods, The MIT Press, Cambridge, pp. 85-104.

Haas, S., Gawande, A. and Reynolds, M.E. (2018), "The risks to patient safety from health system expansions", Journal of the American Medical Association, Vol. 319, p. 1765, doi: 10.1001/jama. 2018.2074

Haas-Wilson, D. and Garmon, C. (2011), "Hospital mergers and competitive effects: two retrospective analyses", International Journal of the Economics of Business, Vol. 18, pp. 17-32, doi: 10.1080/ 13571516.2011.542952.

Harris, J., Ozgen, H. and Ozcan, Y. (2000), "Do mergers enhance the performance of hospital efficiency?", Journal of the Operational Research Society, Vol. 51, pp. 801-811, doi: 10.1057/palgrave.jors.2600869.

Hayford, T.B. (2012), "The impact of hospital mergers on treatment intensity and health outcomes", Health Services Research, Vol. 47, pp. 1008-1029, doi: 10.1111/j.1475-6773.2011.01351.x.

Helderman, J.K., Schut, F.T., van der Grinten, T.E.D. and van de Ven, W.P.M.M. (2005), "Marketoriented health care reforms and policy learning in The Netherlands", Journal of Health Politics Policy and Law, Vol. 30, pp. 189-210, doi: 10.1215/03616878-30-1-2-189.

Ho, V. and Hamilton, B.H. (2000), "Hospital mergers and acquisitions: does market consolidation harm patients?", Journal of Health Economics, Vol. 19, pp. 767-791, doi: 10.1016/S0167-6296(00) 00052-7.

Hutchings, A., Allen, P., Fulop, N., King, A., Protopsaltis, G., Normand, C. and Walters, R. (2003), "The process and impact of trust mergers in the national health service: a financial perspective", Public Money Manag, Vol. 23, pp. 103-112, doi: 10.1080/09540962.2003.10874831.

Kjekshus, L. and Hagen, T. (2007), "Do hospital mergers increase hospital efficiency? Evidence from a National Health Service country", Journal of Health Services Research and Policy, Vol. 12, pp. 230-235, doi: 10.1258/135581907782101561.

May, C. and Finch, T. (2009), "Implementing, embedding, and integrating practices: an outline of normalization process theory”, Sociology, Vol. 43, pp. 535-554, doi: 10.1177/0038038509103208.

Mutter, R.L., Romano, P.S. and Wong, H.S. (2011), "The effects of US hospital consolidations on hospital quality", International Journal of the Economics of Business, Vol. 18, pp. 109-126, doi: 10.1080/13571516.2011.542961.

Postma, J. and Roos, A.F. (2016), "Why healthcare providers merge", Health Economics, Policy and Law, Vol. 11, pp. 121-140, doi: 10.1017/S1744133115000304.

Rohde, T. and Torvatn, H. (2017), "A strategic document as a tool for implementing change. Lessons from the merger creating the South-East Health region in Norway", Health Policy, Vol. 121, pp. 525-533, doi: 10.1016/j.healthpol.2017.02.014. 
Romano, P.S. and Balan, D.J. (2011), "A retrospective analysis of the clinical quality effects of the acquisition of highland park hospital by evanston northwestern healthcare", International Journal of the Economics of Business, Vol. 18, pp. 45-64, doi: 10.1080/13571516.2011.542955.

Roos, A.F. (2018), Mergers and Competition in the Dutch Healthcare Sector, Erasmus University Rotterdam, Rotterdam.

Sabel, C., Herrigel, G. and Kristensen, P.H. (2018), "Regulation under uncertainty: the coevolution of industry and regulation: regulation under uncertainty", Regul. Gov, Vol. 12, pp. 371-394, doi: 10. 1111/rego.12146.

Schmid, A. and Ulrich, V. (2013), "Consolidation and concentration in the German hospital market: the two sides of the coin", Health Policy, Vol. 109, pp. 301-310, doi: 10.1016/j.healthpol.2012.08.012.

Schmid, A. and Varkevisser, M. (2016), "Hospital merger control in Germany, The Netherlands and England: experiences and challenges”, Health Policy, Vol. 120, pp. 16-25, doi: 10.1016/j.healthpol. 2015.11.002.

Schut, F.T. and Varkevisser, M. (2017), "Competition policy for health care provision in The Netherlands", Health Policy, Vol. 121, pp. 126-133, doi: 10.1016/j.healthpol.2016.11.002.

Tavory, I. and Timmermans, S. (2014), Abductive Analysis: Theorizing Qualitative Research, The University of Chicago Press, Chicago.

van de Bovenkamp, H.M., de Mul, M., Quartz, J.G.U., JWM Weggelaar-Jansen, A.M. and Bal, R. (2014), "Institutional layering in governing healthcare quality", Public Administration, Vol. 92, pp. 208-223, doi: 10.1111/padm.12052.

Varkevisser, M. and Schut, F.T. (2012), "The impact of geographic market definition on the stringency of hospital merger control in Germany and The Netherlands", Health Economics Policy Law, Vol. 7, pp. 363-381, doi: 10.1017/S1744133112000011.

Vogt, W. and Town, R. (2006), "How has hospital consolidation affected the price and quality of hospital care?", The Synthesis Project no. 9, Robert Wood Johnson Foundation, pp. 1-26.

\section{Corresponding author}

David de Kam can be contacted at: dekam@eshpm.eur.nl

For instructions on how to order reprints of this article, please visit our website:

www.emeraldgrouppublishing.com/licensing/reprints.htm

Or contact us for further details: permissions@emeraldinsight.com 